\title{
Research on Motivation and Effect of the Consumers' Internet Word of Mouth Spreading
}

\author{
Kai Xiong \\ Business school, Jianghan University, Manufacturing Development \& Research Center of Wuhan city circle, Wuhan, Hubei, \\ China (xkyx@sina.com)
}

\begin{abstract}
With the development of the information technology, internet word of mouth has become a very important guidepost of the consumer shopping. As a very important way, the influence of the word of mouth marketing is growing gradually recognized by people. To correctly understand the important role of the word of mouth among consumers, it is necessary to know its motivation and effect. This paper describes the meaning of internet word of mouth, analyzes the motivation of internet word of mouth, and probes the positive effect and negative effect of internet word of mouth giving an example.
\end{abstract}

Keywords-internet word of mouth, motivation, effect

\section{消费者网络口碑传播的动机与效果研究}

\author{
熊凯
}

江汉大学商学院, 武汉城市圈制造业发展研究中心, 武汉, 湖北, 中国

\begin{abstract}
摘 要 随着信息技术的发展, 网络口碑传播已经成为消费者购物的重要指南。作为一种重要方式, 口碑营销的影响已经逐渐被 人们所了解。为了正确理解网络口碑传播在消费者中的重要作用, 有必要对其动机和效果进行研究。这篇论文描述了网络口碑传播的 概念, 分析了网络口碑传播的动机, 并通过实证剖析了网络口碑传播的正效应和负效应。
\end{abstract}

关键词 网络口碑传播, 动机, 效果

\section{1. 引言}

早在二十世纪 40 年代, 对口碑的研究就已经开始了, 可是真正把口碑作为一个重要的营销法则, 是从二十世纪 80 年代才进行的。在重视客户价值研究的基础上, 通过对 现有的营销手段加以整合, 不少学者提出了整合营销沟通 的概念, 其中一个非常重要的理论就是口碑营销 (Terence.A.Ship,2001)。近年来, 随着互联网技术的成熟 与发展, 网络口碑对于传统营销模式的冲击越来越明显。 网络口碑相与传统口碑有相似之处, 但却将口碑的作用放 大了几十倍甚至几百倍。传统口碑对顾客态度、购买行为 的影响效果已被证实, 口碑传播双方多属强联结关系, 对 于口碑接收者而言, 口碑传播者的信息可信度高, 说服力 强（Bansal \& Voyer, 2000）[1]。而网络口碑传播所具备的高 效快速、低投入高回报、以及雪球效应等优点都是传统口 碑无法比拟的。因此, 消费者网络口碑的传播将直接影响 下一个购买者, 影响商家和企业的利益。在互联网背景下,
任何企业如果要生存下去, 就一定要把互联网作为企业发 展的一个重中之重, 而网络口碑传播正是企业在互联网上 开路的先行军和主力军。布庸置疑, 21 世纪谁做好网络口 碑, 谁将赢得未来市场。

\section{2. 网络口碑概述}

\section{1 网络口碑概念}

Newman（2003）将网络口碑沟通定义为两个或者多个 消费者之间以电脑为中介进行的文本交换 [2]。Henning （2004）对网络口碑的理解为: 潜在、实际或者先前的顾 客对产品或者公司的任何正面或负面的评价, 该评价能通 过网络传递给大众群体或者组织 ${ }^{[3]}$ 。Litvin（2008）认为, 网络口碑是信息的交流双方通过互联网技术对产品或服务 的使用、特性等进行交流, 并且所有交流者都是消费者。 尽管学者们对网络口碑的定义并不相同, 但可以看出网络 
口碑包括了 3 个基本特征：（1）交流的主体是消费者;（2） 交流渠道通过互联网进行; (3) 交流的内容是对企业的产 品和服务进行评论和交流。从网络口碑的概念可以看出, 网络口碑是通过互联网进行传播的, 其传播主要是通过电 子邮件、新闻组、在线论坛、电子布告栏 ( BBS)、网络 游戏系统、聊天室 (MSN) 等网络形式进行的。消费者之 间的口碑传播 (Word of Mouth, 简称 WOM) 作为一种重 要的评价行为, 被认为具有强大的营销力量, 口碑传播不 仅仅有利于扩大企业、产品品牌的知名度, 更重要的是它 能极大影响口碑受众的消费行为和态度。Katz 和 Lazarsfeld (1955) 的研究发现, 口碑对消费者转换品牌的影响力是 报刊的 7 倍、人员推销的 4 倍、广播广告的 2 倍, 在促使 消费者态度由否定、中立到肯定的转变过程中, 口碑传播 所起的作用则是广告的 9 倍。在网络条件下, 消费者（口 碑发送者）在网络上张贴文章的行为等同于人与人之间的 口碑沟通行为, 只是信息呈现的形式由声音转换成书写的 形式 (Gelb, 1995)。因此, 网络技术的发展推动了传统口 碑的网络化趋势, 越来越多的消费者通过网络交流来收集 产品信息以便做出消费决策, 网络口碑 (IWOM) 成为非 常普遍的现象。

\section{2 网络口碑的特征}

网络口碑沟通与传统口碑面对面的沟通方式相比, 尽 管都是消费者之间有关产品等信息进行的非正式沟通, 但 是二者又有所区别, 网络口碑的传播特征表现在:（1）传 播的范围更广, 网络口碑打破了地域的限制, 在电脑中介 的环境下人际影响的范围和机会均大大提高 (Subramani,2003);（2）传播源的匿名性, 这种特性一方 面可以保护隐私, 传播者有更大的自由发表言论, 使接收 者获得更多更真实的信息 (Johnson, 2000), 另一方面匿 名性也降低了口碑信息的可信度（Smith，2005）;（3）传 播异步性，网络口碑沟通使个人可以在任何时间收发信息， 沟通双方不需要限定同时接触，传播信息可长时间保留;

（4）传播多样性, 网络形式允许人们可以一个人同许多人 同时交流, 同时又可以通过 EMAIL 等形式进行个体间的私 密交流。从这些特征可以看出, 网络口碑较传统口碑传播 的速度和范围都大大提高, 并且还兼有易于搜索的优点。

\section{3 网络口碑对消费者的作用}

2005 年的苏丹红事件, 2006 年的 SKII 金属含量超标 事件, 2008 年三鹿奶粉事件, 2009 年的丰田召回事件等等, 其影响范围和影响力都是经由互联网的放大作用而呈几何 级数爆发, 让销售者们不得不重视网络口碑的作用, 不得 不关注这些来自网络消费者的声音。
张晓飞和董大海（2010）认为网络口碑对消费者的作用 主要表现在三个方面[4]: (1) 实现情感、经验的共享和交换; (2) 提升群体内地位;（3）促进群体互动。口碑传播扩散 过程也是人们互动的过程, 网络的出现与发展, 不仅加快了 接收者获取口碑信息速度, 减少时间与经济投入, 而且口碑 信息量超前丰富, 内容多样, 更重要的是促进了人们互动的 频率和范围。

\section{3. 消费者网络口碑传播的动机}

\section{1 消费者网络口碑传播动机的含义}

网络口碑具有强大的影响力, 但消费者为什么要传递 口碑以及在什么条件下传递口碑, 这引起了学者们极大的 兴趣。对口碑传播动机的研究最早可以追溯到 Dichter 在 1966 年的研究。Dichter 提出了 4 种正向口碑动机: 产品涉 人、自我涉入、他人涉入、信息涉人。Hennig（2004）在 口碑动机研究基础上, 利用网站论坛探讨顾客产生网络口 碑的动机。他从经济学的效用观点出发, 认为消费者发送 口碑能给自己带来效用, 从获得的效用中可以将网络口碑 的发送动机归纳为 7 种动机，包括信息平台的协助、释放 负面的情绪、关心其他消费者、社会利益、经济性激励、 帮助公司和寻求建议, 并且 Hennig 还进一步指出, 社会利 益动机对消费者访问网站和发表评论的影响最大。

互联网的出现很大程度上改变了人们购物的宏观环 境, 人们足不出户, 就可以购书、买花、订票等。网上购 物时, 一个巨大变化是不仅企业可以以较低成本接触顾客, 而且消费者首次由历来被动地接受营销信息, 变为主动参 与。许多购物网站开辟了顾客反馈系统, 只要轻轻点击, 全世界的个人有机会在 “全球社区” 里发表个人经验和想 法, 交换对产品和服务等的意见，逐步形成 “网上口碑”。

网络口碑实际上是一种在线的反馈系统, 鼓励消费者 对产品质量进行评估, 通过网络技术把每个消费者的评价 集中起来, 把它们作为销售方的信用度公布出来, 逐步形 成销售商的信用评价（赖胜强、朱敏，2009）[5]。典型的 网络口碑有淘宝网、当当、卓越网、易趣网等网上买家反 馈的意见。

\section{2 消费者网络口碑传播的动机分析}

为了更进一步了解消费者进行网络口碑传播的动机, 我们以在淘宝网上购买产品 iphone4 为案例来进行分析:

消费者热衰于在淘宝网上购物, 一是因为淘宝的口碑 过硬, 二是产品价格过硬, 三是质量过硬。由于网上的产 品无法触摸, 产品的质量无法判断, 往往需要有影响力的 第三方中介对产品质量作出评估, 然而这样的操作, 费用 
比较高。网络口碑的出现, 正好提供了一种费用低的第三 方媒介, 减缓了这个问题。但是并不是所有在淘宝网上销 售的产品都能称心如意, 只有通过借鉴其他消费者的评价 以及自己的亲身试验, 才能够知道产品质量的好坏。例如 你最近想在网上购买一个 Iphone4 内存为 $16 \mathrm{G}$ 的手机, 你 首先需要搜索你需要东西的名称: iphone $416 \mathrm{G}$, 于是网页 就会显示会出现 100 页的搜索结果, 可以用两种方法来进 行评估:

第一种方法: 我们按信用来将产品重新排列: 显示出 的是湖北武汉的两金冠的买家。产品的网址如下: http://item.taobao.com/item.htm?id=7671863263。打开网页, 我们会关注到两点, 一是销量, 二是评价。我们看到他们 家 30 天销售的产品为 16 件, 因为这款手机的价格比较高, 所以购买的人数较少。第二步就是看评价, 因为很多时候 即使是金冠卖家, 我们也不一定会在他们家购买, 因为可 能售后不好, 或是质量不好等等因素影响消费者的评价。 从这些评价中随便抽出了 59 个作出分类: 满意和不满意。 我们可以根据消费者在网上的评论中看出他们的满意程 度。排除中间有两个消费者因为邮寄的速度而抱怨, 我们 在剩下的 57 个消费者中, 只有 2 个消费者不满意他们所购 买的产品, 这样, 满意度就为 55/57, 约为 $96.5 \%$, 可以说 满意度是相当之高的, 这样我们在这家店购买产品的几率 还是很大的。

第二种方法: 我们按销量来将产品重新排列, 显示出 的是一家海外代购商, 产品的网址如下: http://item.taobao.com/item.htm?id=6277943411。这家 30 天 的销量是 286 件, 有 99 人参加了评论, 按照如上的方法计 算满意度: 93/99, 约为 $93.9 \%$, 略微低于上家的评价。这 家代购的是日版的 iphone4, 价格便宜很多, 16G 内存的根 据跟新的版本分为两个价格: 3998 元和 4200 元, 相比那 家来说, 我们很有可能会心动。调查结果如下: 一共有 50 个有意愿购买 iphone4 的人参加, 有一大半的人选择了购 买日货, 因为觉得便宜。很多人不愿意购买日本版的原因 是日版的价格虽然便宜, 但是日本代购的不好维修, 售后 服务也不好处理, 所以他们选择还是买国内行货。同时我 们也可以分析为什么大家会愿意在网上评论, 不论是好评 还是差评。这是因为大家对自己买到的产品感到满意所以 愿意对产品作出好的评价, 当对自己买到的产品感到不满 意, 又希望借评论的机会来表达一下自己的不满, 同时也 想打消那些原本想在这家购买的消费者。

为什么大家会选择信誉高和销量高的买家来买, 因为 良好的信誉是质量的保证, 信誉度高说明这个产品的质量 好, 得到大家的一致好评。销量也是一种网络信誉的体现, 因为买的人多, 并不是一窝蜂的突然出现的, 是后买的人
看了先买人的意见, 他们才会决定买与不买。很多买家之 所以愿意进行评论, 是想表达自己的情感和满意度。例如, 有一位消费者说货真价实, 这算是很高的评价了, 意思就 是买的值了, 能在网上买到一个便宜而且货真价值的东西 来说是卖家对买家的负责, 这样的产品就是消费者所需要 的。当然遇到不满的买家, 发表负面的评价时也是在所难 免的, 毕竟大家的价值观、审美观都不同, 对产品的要求 也不一样, 像上面举例说明的负面评价, 其实是为了宣泄 自己的不满, 也是出于关心其他消费者的心理, 说出了自 己的真实感受, 告诉其他消费者他的购后态度以及产品的 质量好坏。

因此可以得出结论, 消费者网络口碑传播的动机主要 是: 感情的表达 (正面情绪和负面情绪); 寻求并关心同类 消费者; 社会责任感; 寻求建议。消费者之所以会受到他 人口碑信息的影响, 是因为作为口碑接收者, 他们并不是 只会被动接收口碑信息, 也会主动去搜寻消费信息。一般 说来, 消费者在做出购物决策之前, 若缺乏相应经验和知 识, 就会通过外部搜寻获取信息, 除了搜寻企业提供的信 息 (如广告信息), 更重要的是向周围亲朋好友或通过网上 评论搜寻相关的产品信息。Briggs 和 Hollis（1997）对消费 者的信息收集进行了研究, 他们认为顾客通过网络进行信 息搜寻是基于个人需求而采取的主动行为, 搜寻的信息会 更加符合搜寻目的, 对于接收到的信息会产生较低的排斥 感, 因而信息更容易影响消费决策。由于消费者的主动搜 寻能强化信息的传播效果, 许多学者对口碑受众的信息搜 寻动机和影响搜寻行为的因素进行了研究。Goldsmith （2006）研究了消费者网上搜寻信息的动机，他将搜寻的 动机归纳为 8 种, 分别是减少风险、模仿他人、寻找低价 格、偶然行为、比较酷、受到其他离线信息的刺激、获得 购物的信息以及比较容易接触信息。对于影响口碑受众搜 寻信息行为的因素, 国内有关学者认为, 主要包括消费者 自身的购买卷入程度、创新性、主观知识和认知欲望, 其 中购买卷入和认知欲望与搜寻动机呈正相关, 而创新性、 主观知识与搜寻动机呈负相关。

\section{4. 消费者网络口碑传播的效果}

\section{1 网络口碑传播效果的含义}

消费者网络口碑传播的效果, 是指消费者在网络上进行 网络口碑传播的结果。据此, 网络口碑传播效果可以定义为 消费者的网络口碑传播所产生的影响、反应和效果。我们将 网络口碑分为正面网络口碑和负面网络口碑。正面网络口碑 也就是消费者对于产品的态度倾向于赞同时, 对产品产生的 正面的评价, 我们称消费者正面网络口碑信息引起的效应为 
正面效应。反之, 我们称消费者负面网络口碑引起的效应为 负面效应。

\section{2 消费者网络口碑传播的正效应}

人们之间进行网络口碑传播不仅仅是信息的需要, 很 多时候也是在寻求情感和社会支持。当消费者关注信息本 身的质量对自己决策的影响时, 信息性影响产生了。当消 费者做出社会接收与期望的口碑行为时, 口碑的规范影响 产生了, 这就意味着网络口碑的影响不仅体现在购买行为 的影响, 还影响到消费者的心理和社会需要。当消费者购 买的产品与期望值相符甚至高出期望值时, 消费者就会很 满意, 他们就会在网上发表一些好的评论, 此时就产生了 正面网络口碑。

我们仍然以 iphone4 的网络评论为例, 当某家店铺的 iphone4 的评价都为好或是满意的评价时, 就会催生一种强 大的说服效果, 这些网络信息可以改变口碑接受者的态度 和行为。消费者之所以能在网络畅所欲言, 是因为他们在 论坛上或是评论中可以选择匿名或者非匿名的方式来表达 自己的想法, 他们的想法不受商家的操控, 这些评述是值 得信任的。其他消费者就会因为大家的一致好评而蜂拥而 至, 都来这家店购买。可以说消费者的正面口碑所带来的 是非常可观的效益。

\section{2 消费者网络口碑传播的负效应}

有句古话说的好: 好事不出门, 坏事传千里。正面口 碑的影响固然重要, 但是往往一条负面口碑的信息可能导 致商家巨大的损失。根据复旦大学金立印教授的研究结果 我们了解到: 在网络环境中, 负面口碑对于消费者购买决 策的影响效果要明显高于正面口碑, 这与以往那些在现实 环境中所得出的研究结果一致。

我们可以看到上面有一位卖家的评语是这样的: 无语, 除了无语还是无语! 买了一个 iphone4 一拿到就是坏的, 找保修找理赔, 整整半个月才难道新机! 我现在都不知道 应该是表扬店家, 还是无语! 我除了愤怒, 已经没有别的 话了!

当我们看到这样的评价时, 我们一定不会想在这家购 买了产品了, 更别说向其他人推荐了。就像眼里容不了沙 子一样, 一点不满意的东西, 都要发泄一些, 并告知其他 人不要在某家购买某产品。由此可见负面口碑的作用影响 有多大。当消费者对消费经验或者产品使用经验不满意时, 甚至不愿意再次消费使用时, 消费者常将自身的这种不愉 快的经验告知给周围的相关群体, 这种负面口碑会对销售 额的增长起阻碍作用。在对销售的影响上, 负面口碑的影
响程度是正面口碑信息的两倍以上（Arndt, 1967）。例如, 在过去的研究中, 意见领袖通常被视为群体之中最具有影 响力的人。然而, 当消费者有不满意的经验时, 他们便会 开始传播他们的负面口碑信息, 此时虽然信息传播者不是 意见领袖, 但他们的意见却能对企业产生不利的影响效果 (Blodgett et a1., 1993)。有研究指出不满意的顾客会将自 身不满意的消费经验告诉 9 个人(TARP，1981)。Herr 等人 （1991）指出, 当消费者对一个产品或是服务产生负面意 见时, 该产品或者服务将直接从消费者的考虑范围内删除。

\section{5. 结论}

网络口碑是随着互联网的发展而逐步兴起和发展的, 一些现代的新型营销手段, 例如博客营销、病毒营销等均 是网络口碑的运用, 毫无疑问, 网络口碑营销成为市场营 销未来发展趋势之一（黄敏学、王峰、谢亭亭, 2010) [6]。 根据前文的分析和研究, 消费者进行网络口碑传播的动机 分为以下四点: 感情的表达 (正面情绪和负面情绪); 寻求 并关心同类消费者; 社会责任感; 寻求建议。而消费者网 络口碑传播的效果主要分为正面效果和负面效果。正面的 评价带来正面的效果, 负面的评价带来负面的效果。负面 的效果远远大于正面的效果, 企业应该重视网络口碑的传 播和发展, 通过网络正负口碑, 发现自身的优缺点, 以此 保住消费者对企业的信用, 并扩大企业的影响力和效益。

\section{参考文献(References)}

[1] Bansal, H.5. and Voyer, P.A.Word-of-Mouth Processes Within a Services Purchase Decision Context. Journal of Service Research, 2000, 3(2):166-177.

[2] Newman, P. J. An investigation of consumer reactions to negative word-of-mouth on the Internet. Doctor of Philosophy Dissertation, University of Illinois at Urbana. 2003.

[3] Hennig-Thurau, Thorsten, Kevin P. Gwinner, Gianfranco Walsh, and Dwayne D. Gremler (2004), "Electronic Word-of-Mouth via Consumer-Opinion Platforms: What Motivates Consumers to Articulate Themselves on the Internet?" Journal of Interactive Marketing, 18 (1), 38-52.

[4] Zhang Xiaofei, Dong Dahai. Strategyies of internet word of mouth, Hardware Technology, 2010,(4) (In Chinese).

[5] Lai Shengqiang, Zhu Min. Internet word of mouth review, Finance and Trade Economics, 2009(6) (In Chinese)

[6] Huang Minxue, Wang Feng, Xie Tingting. A preliminary study on word of mouth spread in the network environment, Journal of Management, 2010(1) (In Chinese) 